\title{
Effectiveness and Safety of Long Duration versus Short Duration Diode Laser Transscleral Cyclophotocoagulation
}

This article was published in the following Dove Press journal: Clinical Ophthalmology

\section{Jonathan Cheuk-hung Chan (D) Shing Chuen Chow $\mathbb{D}$ Jimmy Shiu-ming Lai}

Department of Ophthalmology, University of Hong Kong, Hong Kong
Correspondence: Jonathan Cheuk-hung Chan

Department of Ophthalmology, LKS

Faculty of Medicine, University of

Hong Kong, Room 30I, Block B,

Cyberport 4, Hong Kong

Tel +8523962 I405

Email jonnochan@yahoo.com
Purpose: To compare the efficacy and safety of diode laser transscleral cyclophotocoagulation using either the long duration or short duration protocol.

Methods: Retrospective series of 23 consecutive patients with glaucoma who underwent continuous-wave diode laser transscleral cyclophotocoagulation from August 2016 to July 2018 at a tertiary hospital in Hong Kong. Laser pulse duration for the long and short duration protocols was defined as 3.0-4.0 and 1.5-2.0 s, respectively.

Results: There were 15 male and 8 female Chinese subjects (23 eyes), age 49-90 (71.3 \pm 2.7 ), with 10 subjects that underwent long duration cyclophotocoagulation (power $1239.2 \pm 78.3 \mathrm{~mW}$, spots $13.9 \pm 1.4$ ) and 13 subjects that had short duration cyclophotocoagulation (mean power $1817.3 \pm 85.7 \mathrm{~mW}$, spots $14.4 \pm 1.0$ ). Six months after long and short duration cyclophotocoagulation, intraocular pressure decreased significantly from $29.9 \pm 7.8$ to $21.1 \pm 6.5(\mathrm{p}<0.01)$, and from $35.4 \pm 2.7$ to $24.1 \pm 3.4(\mathrm{p}=0.04)$, respectively, while glaucoma medications decreased significantly by $1.4 \pm 0.5(\mathrm{p}=0.02)$ in the long duration group only. Reduction of medications after short duration cyclophotocoagulation was less and did not reach statistical significance $(0.9 \pm 0.9$, $\mathrm{p}=0.15)$. There was no significant difference of visual deterioration and complication rates.

Conclusion: Both types of cyclophotocoagulation were equally effective in lowering intraocular pressure by 6 months, but the short duration protocol, using higher laser power, was able to achieve a greater and earlier reduction, at 3 months. However, the long duration protocol, using less laser power, appears better at reducing medication requirement by 6 months.

Keywords: cyclodiode, cycloablation, cyclodestruction, cyclodestructive, g-probe

\section{Plain Language Summary}

Diode laser transscleral cyclophotocoagulation using the newer long duration protocol is as effective as the original standard short duration protocol for reducing intraocular pressure, and may be more so for reducing glaucoma medications.

\section{Introduction}

Glaucoma is a leading cause of irreversible blindness worldwide. Its progression to blindness is invariably linked to an intraocular pressure (IOP) which is too high for the optic nerve to tolerate without developing accelerated axonal/nerve fibre damage, and in the vast majority of cases, this is due to impairment of aqueous outflow from increased outflow resistance. ${ }^{1,2}$ Transscleral cyclophotocoagulation utilizing continuous-wave diode laser (TSCP) is a non-incisional procedure for ablating the ciliary 
body epithelium covering the ciliary processes, thereby reducing the aqueous production and resulting in a lowering of the IOP. $^{3}$ Although TSCP is currently the most frequently utilized method of cycloablation, there are other types of transscleral cycloablative procedures such as cyclo-cryocoagulation, ${ }^{4}$ ultrasonic coagulation, ${ }^{5}$ and micropulse diode laser cyclophotocoagulation. ${ }^{6}$ In the current practice, TSCP is generally reserved for patients with limited visual prognosis, despite reports of it being safe and effective for patients with good visual potential. ${ }^{7}$ It has been used for many types of glaucoma including primary and secondary open-angle glaucomas, neovascular glaucoma, and refractory glaucoma. ${ }^{8-10}$ The diode laser beam with a wavelength of $810 \mathrm{~nm}$ (within the infrared spectrum) is able to penetrate non-pigmented tissues like conjunctiva and sclera, to be selectively absorbed by pigmented tissue such as the ciliary body epithelium resulting in thermal coagulation necrosis. ${ }^{11}$ Postoperative complications following TSCP include: inflammation (uveitis), hyphema, early IOP spikes, malignant glaucoma, local conjunctival burn, scleral thinning, hypotony, and phthisis bulbi; with varying effect on the postoperative vision. ${ }^{12,13}$ The laser setting for TSCP has traditionally been fixed at around $2 \mathrm{~s}$ (or sometimes, 1.5 s) in duration, with variable power but typically starting at $1.50-1.75 \mathrm{~W}$ and increasing in $0.15-0.25 \mathrm{~W}$ steps until an audible "pop" is heard by the surgeon, at which stage the power is either continued at that level, or decreased by one step down before continuing. Normally 3-4 quadrants are treated at each session, with 4-7 spots (laser burns) applied per quadrant.

With the recent adoption of the "long duration" ("slow coagulation") protocol, TSCP using the previous laser protocol is now referred to as "short duration" TSCP (SDTSCP), to differentiate it from those using the new, long duration protocol (LDTSCP). In LDTSCP, the laser application duration is increased to $3.5-4.0 \mathrm{~s}$, while the power is reduced and fixed at 1.50 $\mathrm{W}$, or $1.25 \mathrm{~W}$ for dark brown irides. ${ }^{14}$ With these lower power settings, there is usually no audible pops, and no attempt to elicit a pop by increasing the laser power is made. Most of the previous studies on TSCP are based on the SDTSCP protocol, ${ }^{3,7-10}$ or compared minor variations of it, ${ }^{15,16}$ and did not examine the more recently introduced LDTSCP protocol. One study which did examine LDTSCP by comparing the IOP reduction following LDTSCP and SDTSCP, found that both have comparable IOP lowering efficacy but LDTSCP appeared to have greater postoperative inflammation and loss of visual acuity. ${ }^{17}$ It should be noted that this study only included eyes that had received a minimum of 24 laser spots per treatment, although the mean number of spots per treatment for either LDTSCP or SDTSCP was not mentioned. The generalization of this study's findings to other locality is limited by the atypically high number of spots per treatment (generally 18-21 spots, as suggested by manufacturer and reported in most studies) and low power for LDTSCP $(900-1100 \mathrm{~mW}$, while $1250-1500$ $\mathrm{mW}$ is suggested by manufacturer). Finally, the study population was from Saudi Arabia, which may not reflect results from Caucasians or other Asians. With limited available data comparing LDTSCP and SDTSCP, especially in Chinese, we aim to retrospectively compare our local results with the two TSCP protocols in terms of efficacy and safety.

\section{Patients and Method}

A consecutive, retrospective review of the medical records of all patients who have received TSCP at Grantham Hospital, Hong Kong, from August 2016 to July 2018 was conducted after obtaining approval from the governing research and ethics committee, the Institutional Review Board of the University of Hong Kong/Hospital Authority Hong Kong West Cluster ("HKU/HA HKWC IRB"), and in a manner adhering to the tenets of the Declaration of Helsinki. Patient informed consent was waived by the research and ethics committee for accessing the retrospective medical data as all study-related data obtained were anonymized and not traceable to the individual patients. The patients were identified through the laser record logbook kept by the Department of Ophthalmology, while their demographical and clinical details were accessed using the hospital's electronic patient record system. Patients satisfying the inclusion and exclusion criteria were included as subjects for further analysis.

The inclusion criteria are:

- Subject age 18 or above, at the time of TSCP,

- Underwent TSCP between 1st August 2016 to 31st July 2018,

- Post-TSCP follow-up duration of at least 26 weeks; extended to one more subsequent visit if failure criteria (see below) reached at the 6-month visit,

- Baseline (pre-operative) IOP $>21 \mathrm{mmHg}$, while using at least one glaucoma medication in the study (treated) eye. 
The exclusion criteria are:

- Incomplete medical records preventing collection of study data,

- Any glaucoma laser procedure (for example, trabeculoplasty or iridotomy) or surgery of the study eye in the preceding 2 months before TSCP,

- Any pre-existing ocular condition which can mimic post-operative complications following TSCP, such as active uveitis or infection, scleral thinning,

- Laser duration of TSCP did not conform to either SD $(1.5-2.0 \mathrm{~s})$ or LD $(3.0-4.0 \mathrm{~s})$.

For applying the diode laser TSCP, the Oculight SLx machine and G-Probes (IRIDEX, CA, USA) were used in all patients. As this was a retrospective study, there were slight variations between surgeons with regard to the laser settings for both types of TSCP treatment. The outcome from a total of 7 surgeons was included in this study, with 5 having performed LDTSCP and 6 having performed SDTSCP for the study cases.

\section{Primary Outcome}

For overall successful IOP control, failure is defined by any of the following criteria:

- $\mathrm{IOP}>21 \mathrm{mmHg}$ on 2 consecutive visits at 6 months onward,

- Less than $20 \%$ IOP reduction from baseline on 2 consecutive visits at 6 months onward,

- IOP of $5 \mathrm{mmHg}$ or lower, with visually significant hypotony-related complications on 2 consecutive visits after 3 months,

- Need for additional cyclo-destructive procedures, or incisional glaucoma surgery,

- Loss of light perception,

- Increase in number of glaucoma medications after 4 weeks.

Eyes with successful IOP control as defined above, while not using any glaucoma medication, will be considered as complete success, while those still requiring glaucoma medication are defined as qualified successes, with the exception that eyes requiring more glaucoma medications (compared to baseline) 4 weeks after the TSCP procedure are considered as failure. Time to failure was recorded for each study eye.

\section{Secondary Outcome}

Preservation of vision and complication rates of the 2 groups will be compared.

Visual deterioration in this study is defined as a persistent drop in visual acuity for 3 months or more, defined as either:

- BCVA loss of 2 or more Snellen lines, or

- a drop to count fingers, or

- a drop from count fingers to hand movement, or

- a drop from hand movement to light perception, or

- a drop from light perception to no light perception.

The occurrence of serious complications following TSCP, including persistent postoperative uveitis ( $\geq 3$ months), hypotony maculopathy, scleral thinning or perforation, phthisis bulbi, and sympathetic ophthalmia were specifically checked for in the medical records, and documented in this study if present.

Data analysis was performed using Microsoft Excel and Prism. Continuous variables were summarized by using mean and standard deviation, while categorical variables were summarized using the number of observations and percentages. All tests of hypotheses were two-tailed. Statistical significance is considered at the 0.05 level. The mean is expressed as mean \pm S.E.M. Two sample independent $t$ test was used for comparing the mean IOP and glaucoma medication reduction, while Fisher's exact test was used for comparing the proportion of IOP control failures and visual deterioration, between the 2 groups.

\section{Results}

A total of 23 subjects (23 eyes) were included in the study. The overall age ranged from 49 to 90 , with a mean of 71.3 \pm 2.7 . The mean age of subjects for the LD and SD TSCP groups were $71.1 \pm 4.9$ and $71.5 \pm 3.0$, respectively. Overall, there were 15 male $(65.2 \%)$ and 8 female $(34.8 \%)$ subjects, with 9 of $10(90.0 \%)$ and 6 of 13 (46.2\%) being males for the LD and SD TSCP groups, respectively. There were 10 eyes which underwent LDTSCP and 13 eyes which underwent SDTSCP. The indications for TSCP included primary open-angle glaucoma (POAG, 13.0\%), primary angle closure glaucoma (PACG, 21.7\%), refractory open-angle glaucoma (refractory $\mathrm{OAG}, 17.4 \%$ ), neovascular glaucoma (NVG, 21.7\%), silicone oil-related glaucoma $(8.7 \%)$, uveitic glaucoma $(4.3 \%)$, angle recession glaucoma $(4.3 \%)$, and others 
(8.8\%). As expected for eyes undergoing TSCP, the majority had diagnosis associated with poor outcome for filtration surgery, with only $13.0 \%$ of eyes being POAG, the rest being refractory $\mathrm{OAG}, \mathrm{PACG}$ and various types of secondary glaucomas. For the LDTSCP group, 20\% of eyes had POAG, 20\% had NVG, 20\% had refractory OAG, while uveitic glaucoma, PACG, Schwartz Matsuo syndrome-related glaucoma, and angle recession glaucoma each accounted for $10.0 \%$. For the SDTSCP group, 30.8\% of eyes had NVG, 23\% had PACG, 15.4\% had refractory OAG, $15.4 \%$ had silicone oil-related glaucoma, while POAG and ghost cell glaucoma each accounted for 7.7\%. The mean baseline IOP in LDTSCP and SDTSCP was $29.8 \pm 3.8$ and $35.4 \pm 2.7 \mathrm{~mm} \mathrm{Hg}$, respectively.

As this was a retrospective study, there were slight variations between surgeons with regard to the laser settings for both types of TSCP treatment. The outcome from a total of 7 surgeons was included in this study, with 5 having performed LDTSCP and 6 having performed SDTSCP for the study cases. As expected, the mean power used per laser spot was lower in LDTSCP compared to SDTSCP, being $1239.2 \pm$ $78.3 \mathrm{~mW}$ and $1817.3 \pm 85.7 \mathrm{~mW}$, respectively. In general, laser pulse duration was fixed at $1.5-2.0 \mathrm{~s}$ for SDTSCP, while initial power was set at $1000-1800 \mathrm{~mW}$, and increased in $100-250 \mathrm{~mW}$ increments (up to a maximum of $2500 \mathrm{~mW}$ ) until an audible "pop" was heard, at which point power was decreased by $100-250 \mathrm{~mW}$ increments until it is just below the threshold for producing an audible "pop". The rest of the procedure was continued at this sub-threshold power level (mean $1817.3 \pm 85.7$, range $1000-2500 \mathrm{~mW}$ ), for a total of $7-24$ (mean $14.4 \pm 1.0$ ) spots over 270 degrees while sparing the 3 and 9 o'clock positions. If no "pop" was heard even with power at $2500 \mathrm{~mW}$, then the rest of the procedure was completed at that power level. For the LDTSCP group, laser pulse duration is fixed at $3.0-4.0 \mathrm{~s}$, while initial laser power was set at 1250-1650 $\mathrm{mW}$ and continues at this level for the entire procedure if no audible "pop" was heard, but is decreased in 100-250 $\mathrm{mW}$ increments whenever a "pop" is heard (mean 1239.2 \pm 78.3 , range $600-1650 \mathrm{~mW}$ ), for a total of 10-29 (mean $13.9 \pm 1.4$ ) spots over 270 degrees while sparing the 3 and 9 o'clock positions.

Note that 1 subject in the LDTSCP group had reached failure criteria for IOP control (by undergoing additional TSCP with micropulse laser) at 4 months and was excluded from all 6-month analyses (except for the overall survival analysis) which now have 9 LDTSCP subjects compared to 13 SDTSCP subjects $(n=22)$.
In terms of IOP reduction within each group [Figure 1], at the 3-month visit $(\mathrm{n}=23$ ), the mean IOP (in $\mathrm{mm} \mathrm{Hg}$ ) decreased from $29.8 \pm 3.8$ to $24.5 \pm 3.9$ after LDTSCP $(\mathrm{p}$ $=0.25$ ), and from $35.4 \pm 2.7$ to $20.8 \pm 3.3$ after SDTSCP $(\mathrm{p}<0.01)$. It should be noted that after 3 months, compared to SDTSCP, the mean IOP reduction after LDTSCP was relatively modest and not statistically significant. By the 6-month visit $(\mathrm{n}=22)$, both group showed significant decrease of mean IOP from baseline, from $29.9 \pm 7.8$ to $21.1 \pm 3.5(\mathrm{p}<0.01)$ and $35.4 \pm 2.7$ to $24.1 \pm 3.4(\mathrm{p}=$ 0.04) after LDTSCP and SDTSCP, respectively. Both group showed no significant IOP changes from 3 to 6 months after TSCP $(n=22)$, although the IOP continued to decrease gradually during this period after LDTSCP $(22.8 \pm 4.0$ to $21.1 \pm 3.5, \mathrm{p}=0.64)$, while it actually increased in the SDTSCP group $(20.8 \pm 3.3$ to $24.1 \pm$ $3.4, \mathrm{p}=0.42$ ).

Comparing the mean postoperative IOP between the 2 groups at 3 or 6 months revealed no significant differences ( $\mathrm{p}=0.48$ and 0.55 , respectively). The mean IOP reduction at 3-month was $5.3 \pm 4.4$ and $14.5 \pm 4.6$, and at 6-month was $8.7 \pm 1.9$ and $11.3 \pm 4.9$, for LDTSCP and SDTSCP, respectively. There was no statistical significant difference between the 2 groups for mean IOP reduction, either at 3 -month $(p=0.48)$ or 6 -month $(p=0.55)$. There was also no significant difference between the 2 groups for mean IOP changes from 3-month to 6-month, despite a drop of $1.7 \pm 3.4$ in the LDTSCP group compared to a rise of $3.2 \pm$ 3.9 in the SDTSCP group $(\mathrm{p}=0.35)$.

For glaucoma medication use, the mean preoperative medications of the LDTSCP group $(n=9)$ decreased from $4.1 \pm 0.3$ to $2.7 \pm 0.5$ at the 6 -month visit, which was a significant reduction of $1.4 \pm 0.5(\mathrm{p}=0.02)$. The mean preoperative medications of the SDTSCP group $(n=13)$ decreased from $3.2 \pm 0.4$ to $2.3 \pm 0.4$ at the 6-month visit, which was a non-significant reduction of $0.9 \pm 0.9(\mathrm{p}=$ 0.15). There was no statistically significant difference between the 2 groups with regard to the mean reduction in medications at 6-month $(\mathrm{p}=0.39)$. [Figure 2] Two of the 13 subjects $(15.4 \%)$ in the SDTSCP group had required increased glaucoma medications at more than 4 weeks after their TSCP, while none in the LDTSCP group had required increased glaucoma medications by the 6-month visit, although this was not statistically significant $(p=0.49)$. As mentioned, this result may be skewed by the exclusion of 1 subject in the LDTSCP group ( 1 failed) at 6-month. 


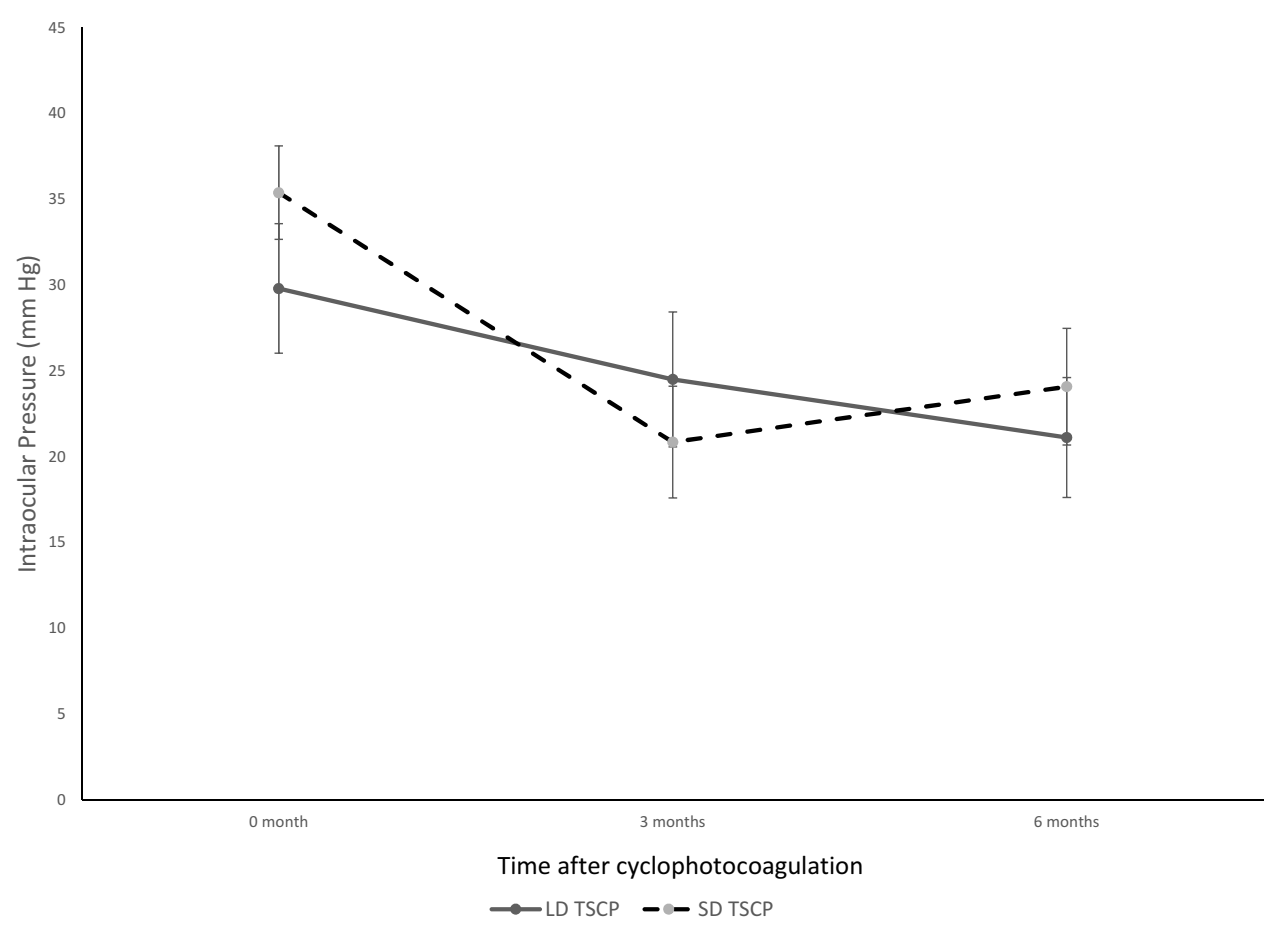

Figure I Mean IOP at baseline, 3 months and 6 months.

Postop 6 months (short duration)

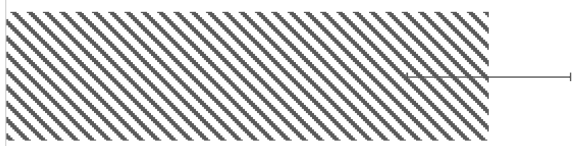

Baseline (short duration)
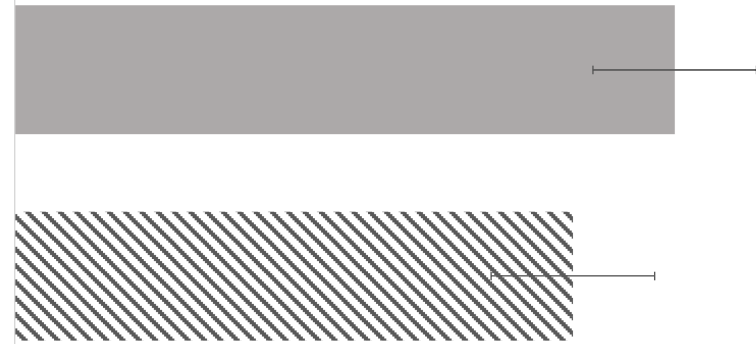

Postop 6 months (long duration)

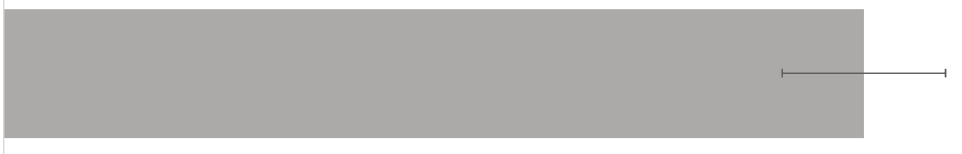

Baseline (long duration)

Figure 2 Mean number of glaucoma medications used at baseline and 6 months.

In terms of successful IOP control at 6-month, 50.0\% (5 of 10) and $61.5 \%$ (8 of 13) of the LDTSCP and SDTSCP groups, respectively, had overall success after a single treatment session. There was no statistically significant difference between the 2 groups in terms of successful IOP control at 6-month $(\mathrm{p}=0.69)$. For the mean time to failure, this was $25.2 \pm 8.6$ and $22.6 \pm 6.7$ weeks for the LDTSCP and SDTSCP groups, respectively, 
with no significant difference between the 2 groups $(\mathrm{P}=$ 0.81). Kaplan-Meier survival analysis of the time to reach failure criteria [Figure 3] also demonstrated no significant difference between the 2 groups $(p=0.72)$.

For visual deterioration by 6-month, one subject from the LDTSCP group was excluded due to having had additional micropulse laser TSCP after the initial LDTSCP treatment (and counted as failure in the IOP control analysis). For the remaining 9 LDTSCP subjects, 3 (33\%) had developed visual deterioration by the 6-month visit, which was comparable to 3 of 13 (23\%) SDTSCP subjects with visual deterioration $(\mathrm{p}=0.66)$. Most of the subjects with visual deterioration in both groups had poor preoperative vision prior to undergoing TSCP, from advanced primary glaucoma or secondary glaucoma (including neovascular glaucoma, or glaucoma following complicated penetrating keratoplasty or retinal detachment surgery).

With regard to complications, no subjects from either group had developed persistent postoperative uveitis, hypotony maculopathy, scleral thinning or perforation, or sympathetic ophthalmia by the 6-month visit. There was one LDTSCP subject with preoperative total rhegmatogenous retinal detachment, Schwartz-Matsuo syndrome and no light perception in the left study eye who developed phthisis bulbi 11 months after TSCP, which is probably unrelated to the cyclophotocoagulation as postoperative
IOP was 22 and 35 (dropped from 53 at baseline) at the 3-month and 6-month visits, respectively.

\section{Discussion}

We had aimed to compare the efficacy and safety of diode laser transscleral cyclophotocoagulation using 2 different treatment protocols in this study. The reason for conducting this retrospective comparative study was the scarcity of reports in the medical literature, at the time of this study, concerning the advantages of the newer protocol of using lower energy and longer duration (LDTSCP) over the previous standard protocol (SDTSCP).

Diode laser transscleral cyclophotocoagulation using either protocol can effectively reduce IOP in our group of mostly refractory or secondary glaucoma subjects. The mean IOP reduction of both treatment groups appears comparable and significantly reduced from baseline at the 6-month visit, although a significant reduction of mean IOP at 3-month was noted only in SDTSCP treated eyes, suggesting that the IOP lowering effect of LDTSCP may be more gradual in onset, compared to SDTSCP. Interestingly, over the entire 6 months study period, the mean IOP trend after LDTSCP showed a modest but progressive reduction, whereas after SDTSCP it showed an earlier and larger reduction followed by a modest recurrent elevation, so that both group's mean IOP are similar

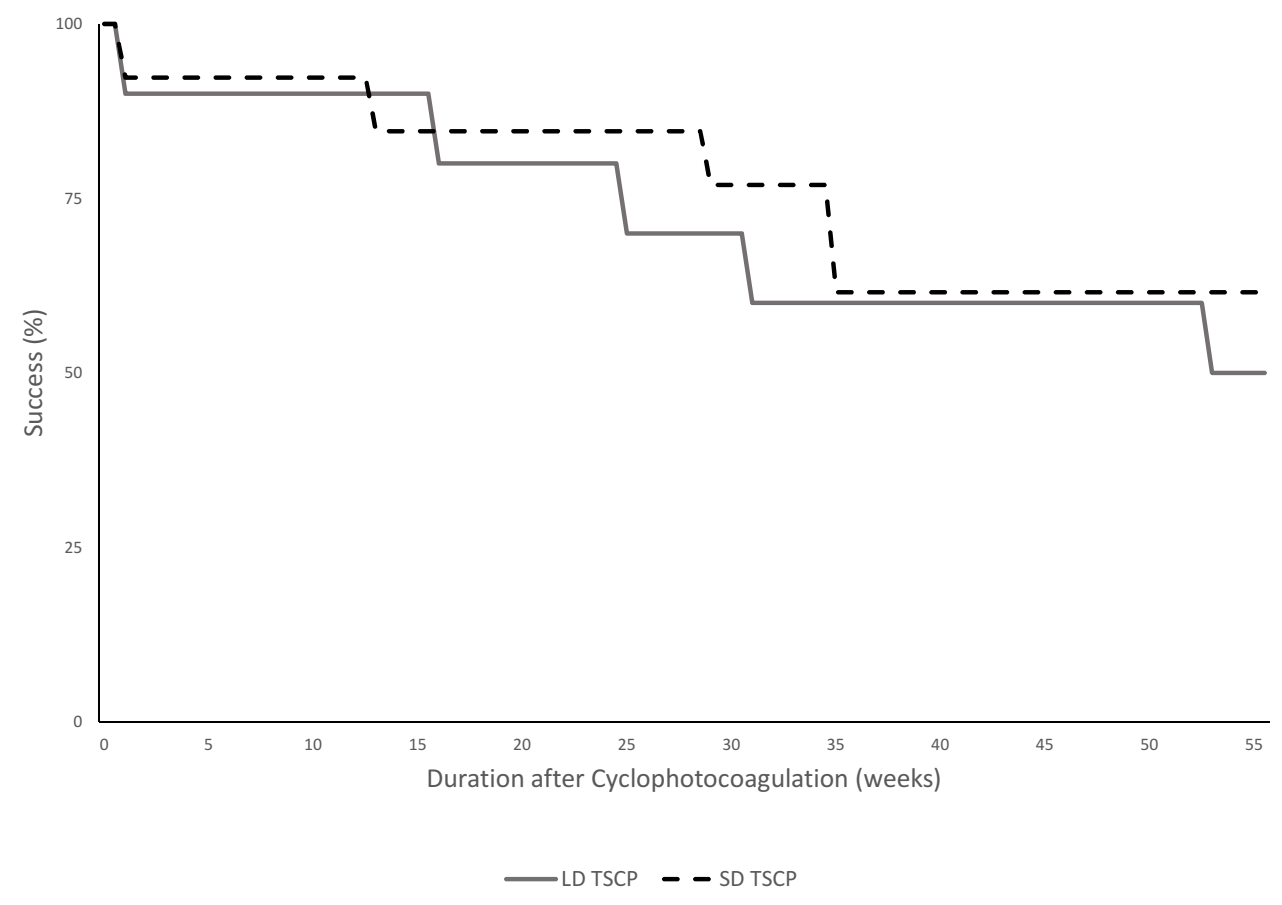

Figure 3 Kaplan-Meier survival curves after TSCP, based on successful IOP control (see definition for primary outcome). 
by 6 months. As TSCP is sometimes used as a temporary intervention for rapid lowering of IOP, before glaucoma surgery can be arranged, ${ }^{18,19}$ it would appear that LSTSCP is less suitable for this indication compared to SDTSCP.

In terms of mean postoperative IOP levels, there was no significant difference shown between the 2 groups, which is to be expected, as most IOP elevations after cyclophotocoagulation will be masked or mitigated with resumption or increase of medications. Therefore, comparing the mean postoperative IOP in isolation, without also considering the extent of glaucoma medication usage, will not be a sensitive method for detecting differences in relative efficacy between the 2 TSCP treatment protocols.

A better indicator of treatment efficacy in our clinical setting would be the glaucoma medication requirement after TSCP, which is typically reduced, similar to any other glaucoma procedures. While both groups in our study had reduced glaucoma medication requirement after TSCP at the 6-month visit, the effect was both greater and statistically significant after LDTSCP (a decrease of 1.5 medications), compared to SDTSCP (a decrease of 0.9 medication) which did not reach statistical significance. There was no significant difference between the 2 groups for mean number of glaucoma medications at the 6-month visit, but $15.4 \%$ of the SDTSCP subjects now required increased (more than baseline) medications, compared to none in the LDTSCP group, although this was not statistically significant. These apparently better results from LDTSCP compared to SDTSCP are despite the slightly lower average number of spots treated during LDTSCP (13.9) compared to SDTSCP (14.4). Our findings suggest LDTSCP may be more effective than SDTSCP in terms of reducing glaucoma medication requirement, with comparable IOP reduction at 6-month being achieved while using less medications. Reduction of topical medications after LDTSCP and SDTSCP can mitigate the local toxic effect of glaucoma drugs and their preservatives on the conjunctiva, and help improve treatment compliance, as well as surgical success of any subsequent subconjunctival aqueous drainage procedures, ${ }^{20-23}$ especially in eyes receiving TSCP as a temporizing treatment.

Due to the small sample size, the study was underpowered for detecting outcome differences between the 2 treatments. Using IOP control success rate, the power was only 0.06 , so an estimated sample size of 841 is required for this to be an acceptably powered ( 0.8 or greater) study. The power is higher $(0.22)$ if we compare medication reduction at 6 months after treatment, so an estimated sample size of 80 should suffice. If comparing the likelihood of requiring increased (more than baseline) medications at 6-month (LDTSCP 0\%, SDTSCP 15.4\%), then the power of this study is 0.27 , and the estimated sample size can be further reduced to 44 .

Another factor affecting IOP control success may be the low number of laser spots used, with both group having a similarly lower mean number of spots per treatment (14.4 spots for SDTSCP; 13.9 spots for LDTSCP) compared to that suggested by the laser manufacturer (18-21 spots).

With regard to the other outcomes, our study did not find any significant differences in visual loss, overall IOP control success rate, time to failure, or complication rates between the 2 treatments. This may be due to the small sample size, and the rarity of serious complications with current cyclophotocoagulation techniques.

\section{Conclusion}

Diode laser transscleral cyclophotocoagulation can significantly lower the intraocular pressure for up to 6 months without serious complications, even in eyes with refractory or secondary glaucomas which are poor candidates for filtration surgery. This effect was noted whether using the old protocol of shorter duration and higher power, or the new protocol with longer duration but lower power. In addition to using lower mean laser power, the long duration treatment protocol also appears to be more effective for reducing postoperative glaucoma medications requirement, albeit having a possibly slower onset of maximal effect and less suitable as a temporizing treatment for severely elevated IOP, but these findings would need to be confirmed with a larger, randomized, prospective study with a longer follow-up period.

\section{Author Contributions}

All authors contributed to data analysis, drafting or revising the article, gave final approval of the version to be published, and agree to be accountable for all aspects of the work. In addition, Chan and Lai had substantial involvement with study concept and design, Chow and Chan had substantial involvement with data acquisition, analysis and interpretation, as well as manuscript drafting.

\section{Disclosure}

The authors have no financial interests or conflicts of interest to report for this work. 


\section{References}

1. Virno M, Sampaolesi R, Pecori Giraldi J, et al. Ibopamine: D1-dopaminergic agonist in the diagnosis of glaucoma. J Glaucoma. 2013;22(1):5-9. doi:10.1097/IJG.0b013e3182311db4

2. Andrew NH, Akkach S, Casson RJ. A review of aqueous outflow resistance and its relevance to microinvasive glaucoma surgery. Surv Ophthalmol. 2020;65(1):18-31. doi:10.1016/j.survophthal.2019.08.002

3. Kosoko O, Gaasterland DE, Pollack IP, Enger CL. Long-term outcome of initial ciliary ablation with contact diode laser transscleral cyclophotocoagulation for severe glaucoma. The Diode Laser Ciliary Ablation Study Group. Ophthalmology. 1996;103(8):1294-1302. doi:10.1016/S0161-6420(96)30508-3

4. Gorsler I, Thieme H, Meltendorf C. Cyclophotocoagulation and cyclocryocoagulation as primary surgical procedures for open-angle glaucoma. Graefes Arch Clin Exp Ophthalmol. 2015;253 (12):2273-2277. doi:10.1007/s00417-015-3159-Z

5. Aptel F, Denis P, Rouland JF, Renard JP, Bron A. Multicenter clinical trial of high-intensity focused ultrasound treatment in glaucoma patients without previous filtering surgery. Acta Ophthalmol. 2016;94(5):e268-e277. doi:10.1111/aos.12913

6. Nguyen AT, Maslin J, Noecker RJ. Early results of micropulse transscleral cyclophotocoagulation for the treatment of glaucoma. Eur J Ophthalmol. 2019;2:1120672119839303. doi:10.1177/1120672119839303

7. Shah P, Bhakta A, Vanner EA, Kishor KS, Greenfield DS, Maharaj ASR. Safety and efficacy of diode laser transscleral cyclophotocoagulation in eyes with good visual acuity. J Glaucoma. 2018;27(10):874-879. doi:10.1097/IJG.0000000000001057

8. Ansari E, Gandhewar J. Long-term efficacy and visual acuity following transscleral diode laser photocoagulation in cases of refractory and non-refractory glaucoma. Eye (Lond). 2007;21(7):936-940. doi:10.1038/sj.eye.6702345

9. Choy BNK, Lai JSM, Yeung JCC, Chan JCH. Randomized comparative trial of diode laser transscleral cyclophotocoagulation versus Ahmed glaucoma valve for neovascular glaucoma in Chinese a pilot study. Clin Ophthalmol. 2018;7(12):2545-2552. doi:10.2147/ OPTH.S188999

10. Cheung JJC, Li KKW, Tang SWK. Retrospective review on the outcome and safety of transscleral diode laser cyclophotocoagulation in refractory glaucoma in Chinese patients. Int Ophthalmol. 2019;39 (1):41-46. doi:10.1007/s10792-017-0782-1

11. Assia EI, Hennis HL, Stewart WC, Legler UF, Carlson AN, Apple DJ. A comparison of neodymium: yttrium aluminum garnet and diode laser transscleral cyclophotocoagulation and cyclocryotherapy. Invest Ophthalmol Vis Sci. 1991;32(10):2774-2778.
12. Ishida K. Update on results and complications of cyclophotocoagulation. Curr Opin Ophthalmol. 2013;24(2):102-110. doi:10.1097/ ICU.0b013e32835d9335

13. Quigley HA. Improved outcomes for transscleral cyclophotocoagulation through optimized treatment parameters. J Glaucoma. 2018;27 (8):674-681. doi:10.1097/IJG.0000000000001008

14. IRIDEX corporation webpage information for physicians. Available from: https://www.iridex.com/PhysicianEducation/Glaucoma/ TSCPCG-Probe.aspx. Accessed January 7, 2020

15. Chang SH, Chen YC, Li CY, Wu SC. Contact diode laser transscleral cyclophotocoagulation for refractory glaucoma: comparison of two treatment protocols. Can J Ophthalmol. 2004;39(5):511-516. doi:10.1016/S0008-4182(04)80140-6

16. Stevenson-Fernandez MO, Rodriguez-Garcia A, Espino-Barros Palau A, Gonzalez-Madrigal PM. Efficacy and safety of pop-titrated versus fixed-energy trans-scleral diode laser cyclophotocoagulation for refractory glaucoma. Int Ophthalmol. 2019;39(3):513-519. doi:10.1007/s10792-018-0834-1

17. Alzuhairy S, Albahlal A, Aljadaan I, et al. Intraocular pressure outcomes following transscleral diode cyclophotocoagulation using long and short duration burns. J Glaucoma. 2016;25(9):e782-6. doi:10.1097/ IJG.0000000000000503

18. Weinreb RN, Crowston JG. WGA Consensus Series 2: Glaucoma Surgery - Open Angle Glaucoma. Kugler Publications; 2005.

19. Kirwan JF, Shah P, Khaw PT. Diode laser cyclophotocoagulation: role in the management of refractory pediatric glaucomas. Ophthalmology. 2002;109(2):316-323. doi:10.1016/S0161-6420(01) 00898-3

20. Broadway DC, Grierson I, O'Brien C, Hitchings RA. Adverse effects of topical antiglaucoma medication. I. The conjunctival cell profile. Arch Ophthalmol. 1994;112(11):1437-1445. doi:10.1001/archopht.1994.0109 0230051020

21. Broadway DC, Grierson I, O'Brien C, Hitchings RA. Adverse effects of topical antiglaucoma medication. II. The outcome of filtration surgery. Arch Ophthalmol. 1994;112(11):1446-1454. doi:10.1001/ archopht.1994.01090230060021

22. Leng F, Liu P, Li H, Zhang J. Long-term topical antiglaucoma medications cause enhanced Tenon's capsule fibroblast proliferation and abnormal TGF- $\beta$ and MMP expressions: potential effects on glaucoma filtering surgery. Curr Eye Res. 2011;36(4):301-309. doi:10.3109/02713683.2010.547648

23. Mastropasqua R, Mastropasqua R, Fasanella V, et al. In vivo confocal imaging of the conjunctiva as a predictive tool for the glaucoma filtration surgery outcome. Invest Ophthalmol Vis Sci. 2017;58(6): BIO114-BIO120. doi:10.1167/iovs.17-21795
Clinical Ophthalmology

\section{Publish your work in this journal}

Clinical Ophthalmology is an international, peer-reviewed journal covering all subspecialties within ophthalmology. Key topics include: Optometry; Visual science; Pharmacology and drug therapy in eye diseases; Basic Sciences; Primary and Secondary eye care; Patient Safety and Quality of Care Improvements. This journal is indexed on PubMed
Central and CAS, and is the official journal of The Society of Clinical Ophthalmology (SCO). The manuscript management system is completely online and includes a very quick and fair peer-review system, which is all easy to use. Visit http://www.dovepress.com/ testimonials.php to read real quotes from published authors. 